\title{
Chaotic behavior and damage spreading in the Glauber Ising model - a master equation approach
}

\author{
Thomas Vojta \\ Institut für Physik, Technische Universität, D-09107 Chemnitz, Germany and \\ Department of Physics and Materials Science Institute, University of Oregon, Eugene, OR97403, USA
}

(March 29, 2022)

\begin{abstract}
We investigate the sensitivity of the time evolution of a kinetic Ising model with Glauber dynamics against the initial conditions. To do so we apply the "damage spreading" method, i.e., we study the simultaneous evolution of two identical systems subjected to the same thermal noise. We derive a master equation for the joint probability distribution of the two systems. We then solve this master equation within an effective-field approximation which goes beyond the usual mean-field approximation by retaining the fluctuations though in a quite simplistic manner. The resulting effective-field theory is then applied to different physical situations. It is used to analyze the fixed points of the master equation and their stability and to identify regular and chaotic phases of the Glauber Ising model. We also discuss the relation of our results to directed percolation.
\end{abstract}

PACS numbers: 05.40.+j, 64.60.Ht, 75.40.Gb

\section{INTRODUCTION}

The physics of dynamic phase transitions and dynamic critical phenomena has been a subject of great interest for the last two decades. Whereas the dynamic behavior at and close to usual static phase transitions is well understood [1,2] much less is known about dynamic phase transitions which do not have a static counterpart. Sometimes it is not even known whether or not a particular dynamic transition coincides with an equilibrium phase transition.

One of these dynamic phenomena is the so-called "damage spreading" [3 5]. The central question of this problem is how a small perturbation (called the damage) in a cooperative system changes during the further time evolution. Among the simplest of such cooperative systems are kinetic Ising models where the above question has been investigated by means of Monte-Carlo simulations [4, 5]. In these simulations two identical Ising models with different initial conditions are subjected to the same thermal noise, i.e. the same random numbers are used in the Monte-Carlo procedure. In analogy to the physics of chaotic dynamics [6] the differences in the microscopic configurations of the two systems are then used to characterize the dynamics and to distinguish regular and chaotic phases, depending on external parameters (e.g. temperature, magnetic field).

Later the name "damage spreading" has also been applied to a different though related type of investigations in which the two systems are not identical. Instead, one or several spins in one of the copies are permanently fixed in one direction. Therefore the equilibrium properties of the two systems are different and the microscopic differences between the two copies can be related to static and dynamic correlation functions [7,8]. Note that in this type of simulations it is not essential to use identical noise (i.e. random numbers) for the two systems. Instead it is only a convenient method to reduce the statistical error.

Whereas this second type of damage spreading is well understood and established as a method to numerically calculate equilibrium properties, much less is known about the original problem of damage spreading, viz. how sensitive is the dynamics of the Ising model to different initial conditions. In particular, there are no rigorous results on the transition between regular and chaotic behavior (called the "spreading transition").

There are two different mechanisms by which the damage can spread in a kinetic Ising model. First, the damage can spread within a single ergodic component (i.e. a pure state or free energy valley) of the system. This is the case for Glauber or Metropolis dynamics. Numerical simulations here consistently give a transition temperature slightly lower than the equilibrium critical temperature [9]. Grassberger [10] conjectured that the spreading transition falls into the universality class of directed percolation if it does not coincide with another phase transition. This was supported by high-precision numerical simulations for the Glauber Ising model [11].

Second, the damage can spread when the system selects one of the free energy valleys at random after a quench from high temperatures to below the equilibrium critical temperature. This is the only mechanism to produce damage spreading in an Ising model with heat-bath dynamics. In this case the spreading temperature seems to coincide with the equilibrium critical temperature below which the two pure states separate [4, 12,13]. Thus, at the spreading point there are long-range static correlations in the systems, and the transition is expected to fall into a universality class different from directed percolation.

In this paper we investigate the damage spreading in the Glauber Ising model by deriving and solving a master equation for the time evolution of a joint probability distribution for two identical systems with different initial conditions and subjected to the same thermal noise. The paper is organized as follows. In Section IIA we define the model. Transition probabilities between the states of a spin pair are calculated in section IIB and 
the master equation for the joint probability distribution is derived in section IIC. We discuss how to construct a mean-field approximation for this equation in section IIIA. In sections IIIB, IIIC and IIID we present solutions of the master equation within this approximation for different physical situations. Finally, section IV is dedicated to conclusions and an outlook on future work. A short account of part of this work has already been published [14] together with a comparison to the heat-bath Ising model.

\section{A MASTER EQUATION FOR DAMAGE SPREADING}

\section{A. The Glauber Ising model}

We consider two identical kinetic Ising models with $N$ sites described by the Hamiltonians $H^{(1)}$ and $H^{(2)}$ given by

$$
H^{(n)}=-\frac{1}{2} \sum_{i j} J_{i j} S_{i}^{(n)} S_{j}^{(n)}-h \sum_{i} S_{i}^{(n)}
$$

where $S_{i}^{(n)}$ is an Ising variable with the values \pm 1 and $n=1,2$ distinguishes the two copies. $J_{i j}$ is the exchange interaction between the spins and $h$ denotes an external magnetic field. The dynamics of the Ising models is given by the Glauber algorithm, i.e. in every time step a lattice site $i$ is chosen at random (the same site in both copies). The new value of the spin at this site is calculated according to

$$
S_{i}^{(n)}(t+1)=\operatorname{sign}\left\{v\left[h_{i}^{(n)}(t)\right]-\frac{1}{2}+S_{i}^{(n)}(t)\left[\xi_{i}(t)-\frac{1}{2}\right]\right\}
$$

where the transition probality $v(x)$ is given by the usual Glauber expression

$$
v(x)=e^{x / T} /\left(e^{x / T}+e^{-x / T}\right) .
$$

Here $h_{i}^{(n)}(t)=\sum_{j} J_{i j} S_{j}^{(n)}(t)+h$ is the local magnetic field at site $i$ and (discretized) time $t$ in the system $n$. $\xi_{i}(t) \in[0,1)$ is a random number which is identical for both systems, and $T$ denotes the temperature. The spins at all sites different from site $i$ are unchanged within this time step.

The central quantity in any damage spreading process is the distance between the two systems in phase space, called the Hamming distance (or the damage). It is defined by

$$
D(t)=\frac{1}{2 N} \sum_{i=1}^{N}\left|S_{i}^{(1)}(t)-S_{i}^{(2)}(t)\right|
$$

and identical to the portion of sites where the spins in the two systems differ.
In order to describe the simultaneous time evolution of the two systems $H^{(1)}$ and $H^{(2)}$ we define a variable $\nu(t)$ at each lattice site which describes the state of a spin pair $\left(S^{(1)}, S^{(2)}\right)$. It has the values $\nu=++$ for $S^{(1)}=$ $S^{(2)}=1$, +- for $S^{(1)}=-S^{(2)}=1,-+$ for $-S^{(1)}=$ $S^{(2)}=1$ and -- for $S^{(1)}=S^{(2)}=-1$. A complete configuration of the two Ising models is thus described by the set $\left\{\nu_{1}, \ldots, \nu_{N}\right\}$.

Since we are interested in the time evolution not for a single sequence of $\xi_{i}(t)$, but in $\xi$-averaged quantities we consider a whole ensemble of system pairs $\left(H^{(1)}, H^{(2)}\right)$ and define a probability distribution

$$
P\left(\nu_{1}, \ldots, \nu_{N}, t\right)=\left\langle\sum_{\nu_{i}(t)} \prod_{i} \delta_{\nu_{i}, \nu_{i}(t)}\right\rangle
$$

where $\langle\cdot\rangle$ denotes the ensemble average.

\section{B. Transition probabilities}

In order to formulate a master equation for the probability distribution $P\left(\nu_{1}, \ldots, \nu_{N}, t\right)$ we need to know the transition probabilities $w(\nu \rightarrow \mu)$ between the states $\nu$ of a spin pair. Since the Glauber dynamics (2) involves only a single lattice site within each time step, we have to consider transitions between the states $\nu$ of a single site only. Let us look, e.g., at the transition of site $i$ from state -to ++ . This corresponds to both $S^{(1)}$ and $S^{(2)}$ changing from -1 to 1 . According to the Glauber dynamics (2) this requires $v\left(h_{i}^{(1)}\right)-\xi_{i}>0$ and $v\left(h_{i}^{(2)}\right)-\xi_{i}>0$. Since $v(h)$ is a monotonous function of $h$ both equations are simultaneously fulfilled for $v\left[\min \left(h_{i}^{(1)}, h_{i}^{(2)}\right)\right]-\xi_{i}>0$. Because $\xi_{i}$ is a random number taken from a uniform distribution between 0 and 1, the transition probability is given by

$$
w(--\rightarrow++)=v\left[\min \left(h^{(1)}, h^{(2)}\right)\right] .
$$

Analogously, for a transition from state -- to +- the two inequalities $v\left(h_{i}^{(1)}\right)-\xi_{i}>0$ and $v\left(h_{i}^{(2)}\right)-\xi_{i}<0$ have to be fulfilled. Since $v(h)$ is a monotonous function of $h$ this is only possible for $h_{i}^{(1)}>h_{i}^{(2)}$. The transition probability is obviously given by

$$
w(--\rightarrow+-)=\Theta\left(h^{(1)}-h^{(2)}\right)\left[v\left(h^{(1)}\right)-v\left(h^{(2)}\right)\right] .
$$

The transition probabilities $w(\nu \rightarrow \mu)$ fulfill the following symmetry relations

$$
\begin{aligned}
& w(++\rightarrow \nu)=w(--\rightarrow \nu) \\
& w(+-\rightarrow \nu)=w(-+\rightarrow \nu)
\end{aligned}
$$

for any state $\nu$ as can easily be seen by making the substitutions $S_{i}^{(n)}(t) \rightarrow-S_{i}^{(n)}(t)$ and $\xi_{i}(t) \rightarrow 1-\xi_{i}(t)$ on the right-hand side of $(2)$.

The remaining transition probabilities can be calculated along the same lines as above. They are summarized in Table I. 
TABLE I. Transition probabilities $w(\nu \rightarrow \mu)$ between the states of a spin pair

\begin{tabular}{lc}
\hline \hline$--\rightarrow--$ & $v\left[-\max \left(h^{(1)}, h^{(2)}\right)\right]$ \\
$--\rightarrow++$ & $v\left[\min \left(h^{(1)}, h^{(2)}\right)\right]$ \\
$--\rightarrow-+$ & $\Theta\left(h^{(2)}-h^{(1)}\right)\left[v\left(h^{(2)}\right)-v\left(h^{(1)}\right)\right]$ \\
$--\rightarrow+-$ & $\Theta\left(h^{(1)}-h^{(2)}\right)\left[v\left(h^{(1)}\right)-v\left(h^{(2)}\right)\right]$ \\
\hline$-+\rightarrow--$ & $\Theta\left(-h^{(1)}-h^{(2)}\right)\left[v\left(-h^{(1)}\right)-v\left(h^{(2)}\right)\right]$ \\
$-+\rightarrow++$ & $\Theta\left(h^{(1)}+h^{(2)}\right)\left[v\left(h^{(2)}\right)-v\left(-h^{(1)}\right)\right]$ \\
$-+\rightarrow-+$ & $v\left[\min \left(-h^{(1)}, h^{(2)}\right)\right]$ \\
$-+\rightarrow+-$ & $v\left[-\max \left(-h^{(1)}, h^{(2)}\right)\right]$ \\
\hline \hline
\end{tabular}

\section{The master equation}

Having calculated the transition probabilities between the states $\nu$ of a spin pair we are now in the position to write down the equation of motion for the probalitity distribution $P\left(\nu_{1}, \ldots \nu_{N}, t\right)$. It has the form of a usual master equation

$$
\begin{aligned}
& \frac{d}{d t} P\left(\nu_{1}, \ldots, \nu_{N}, t\right)= \\
& -\sum_{i=1}^{N} \sum_{\mu_{i} \neq \nu_{i}} P\left(\nu_{1}, \ldots, \nu_{i}, \ldots, \nu_{N}, t\right) w\left(\nu_{i} \rightarrow \mu_{i}\right) \\
& +\sum_{i=1}^{N} \sum_{\mu_{i} \neq \nu_{i}} P\left(\nu_{1}, \ldots, \mu_{i}, \ldots, \nu_{N}, t\right) w\left(\mu_{i} \rightarrow \nu_{i}\right)
\end{aligned}
$$

where the term in the second line describes the decrease of $P\left(\nu_{1}, \ldots \nu_{N}, t\right)$ due to the initial configuration $\left\{\nu_{1}, \ldots \nu_{N}\right\}$ being changed at one of the sites $i$ from $\nu_{i}$ to $\mu_{i}$. The term in the third line of the master equation describes the increase of $P\left(\nu_{1}, \ldots \nu_{N}, t\right)$ due to "scattering" from all the other states into $\left\{\nu_{1}, \ldots \nu_{N}\right\}$. Note that we have suppressed the factor $1 / N$ in the transition probabilities which corresponds to random selection of one of the lattice sites in every time step. This neglect corresponds to a redefinition of the time scale (which is now independent of the system size) and does not change the dynamic behavior.

This master equation contains, of course, the full difficulty of the dynamic many-body problem. A complete solution is therefore out of question, and one has to resort to approximation methods. In the following section we discuss how to construct a mean-field like approximation to the master equation (9).

\section{EFFECTIVE-FIELD APPROXIMATION}

Usually a mean-field theory of a phase transition can be obtained by taking the range of the interaction to infinity:

$$
J_{i j}=J_{0} / N \quad \text { for all } i, j
$$

In the thermodynamic limit $N \rightarrow \infty$ this suppresses all fluctuations. In particular, the local magnetic fields $h_{i}^{(n)}$ of all sites in one system become equal and identical to the mean-field value $J_{0} m$. Since the two Ising models $H^{(1)}$ and $H^{(2)}$ are thermodynamically identical this leads to $h_{i}^{(1)}=h_{i}^{(2)}$. However, some of the transition probabilities depend on the existence of fluctuations (see table I), i.e. $w(\nu \rightarrow \mu)$ go to zero with $h^{(1)}-h^{(2)} \rightarrow 0$. In particular, this is true for $w(--\rightarrow-+)$ and $w(--\rightarrow$ $+-)$ which are responsible for increasing the damage $D$. Consequently, if the thermodynamic limit and the limit of infinite range of the interaction are taken at a too early stage of the calculation, the resulting model does not show any spreading of the damage. To circumvent these problems we develop a slightly more sophisticated effective-field approximation that retains the fluctuations though in a quite simplistic manner. As will be shown in section IIIC, taking the range of the interaction to infinity within the framework of this approximation yields a sensible limit.

\section{A. Effective-field theory for short-range models}

The central idea of this effective-field method is to retain the fluctuations but to treat the fluctuations at different sites as statistically independent. This amounts to approximating the probability distribution $P\left(\nu_{1}, \ldots, \nu_{N}, t\right)$ by a product of identical single-site distributions $P_{\nu}$

$$
P\left(\nu_{1}, \ldots, \nu_{N}, t\right)=\prod_{i=1}^{N} P_{\nu_{i}}(t) .
$$

Inserting this into the master equation (9) and summing over all states of sites $i=2 \ldots N$ gives an equation of motion for the single-site distribution $P_{\nu_{1}}$,

$$
\frac{d}{d t} P_{\nu_{1}}=\sum_{\mu_{1} \neq \nu_{1}}\left[-P_{\nu_{1}} W\left(\nu_{1} \rightarrow \mu_{1}\right)+P_{\mu_{1}} W\left(\mu_{1} \rightarrow \nu_{1}\right)\right]
$$

where

$$
W\left(\mu_{1} \rightarrow \nu_{1}\right)=\left\langle w\left(\mu_{1} \rightarrow \nu_{1}\right)\right\rangle_{P}
$$

is the transition probability averaged over the states $\nu_{i}$ of all sites $i \neq 1$ according to the distribution $P_{\nu_{i}}$. Since all sites of the systems are equivalent the site index $i$ will be suppressed from now on.

Note that the average magnetizations $m^{(1)}, m^{(2)}$ of the two systems and the Hamming distance $D$ can be easily expressed in terms of $P_{\nu}$,

$$
\begin{aligned}
m^{(1)} & =P_{++}+P_{+-}-P_{-+}-P_{--}, \\
m^{(2)} & =P_{++}-P_{+-}+P_{-+}-P_{--}, \\
D & =P_{+-}+P_{-+} .
\end{aligned}
$$


TABLE II. Probabilities for the states of the three neighboring sites and resulting local magnetic fields $h^{(1)}$ and $h^{(2)}$ for the two-dimensional case on the hexagonal lattice

\begin{tabular}{ccccc}
\hline \hline & \multicolumn{4}{c}{$h^{(1)}$} \\
$h^{(2)}$ & $3 J$ & $J$ & $-J$ & $-3 J$ \\
\hline $3 J$ & $P_{++}^{3}$ & $3 P_{++}^{2} P_{-+}$ & $3 P_{++} P_{-+}^{2}$ & $P_{-+}^{3}$ \\
$J$ & $3 P_{++}^{2} P_{+-}$ & $3 P_{++}^{2} P_{--}+$ & $3 P_{+-} P_{-+}^{2}+$ & $3 P_{-+} P_{--}^{2}$ \\
& & $6 P_{++} P_{+-} P_{-+}$ & $6 P_{+-} P_{-+} P_{--}$ \\
$-J$ & $3 P_{++} P_{+-}^{2}$ & $3 P_{+-}^{2} P_{-+}+$ & $3 P_{++} P_{--}^{2}+$ & $3 P_{-+} P_{--}^{2}$ \\
& \multicolumn{5}{c}{$6 P_{++} P_{+-} P_{--}$} & $6 P_{+-} P_{-+} P_{--}$ & \\
$-3 J$ & $P_{+-}^{3}$ & $3 P_{+-}^{2} P_{--}$ & $3 P_{+-} P_{--}^{2}$ & $P_{--}^{3}$ \\
\hline \hline
\end{tabular}

So far the considerations have been rather general, in the following subsections we will apply the general formalism to different physical situations. In section IIIB we investigate a two-dimensional system with short-range interactions and vanishing external field. We determine not only the location of the spreading transition but also calculate the stationary states of the systems. Section IIIC deals with the limit of infinite-range interactions, and in sec. IIID we study the influence of an external magnetic field on the spreading transition.

\section{B. Solution of a two-dimensional model}

In this subsection we investigate the damage spreading for a two-dimensional Glauber Ising model on a hexagonal lattice (with each site having three nearest neighbors). The interaction is taken to be a nearest-neighbor interaction of strength $J$ and the external magnetic field is set to zero.

In order to solve the master equation (12) for the single-site distribution $P_{\nu}$ we first determine the effective transition probabilities $W(\nu \rightarrow \mu)$. Let us calculate the probabilities for a particular site $i$. To this end we have to average the transition probabilities given in table I with respect to the states of all other sites of the system. However, since the interaction is between nearest neighbors, the transition probabilities depend on the states of these three neighbors of site $i$ only. Each of the neighbors can be in one of four states, thus we have to consider 64 different configurations of the neighboring sites. The probabilities for these configurations and the resulting local magnetic fields are given in table II.

With the help of tables I and II the averaged transition probabilities (13) can be easily calculated by adding up the contributions of all 64 configurations. The resulting expression are quite lengthy though simple. Therefore we present only the example

$$
\begin{aligned}
& W(--\rightarrow++)= \\
& \quad\langle w(--\rightarrow++)\rangle=\left\langle v\left[\min \left(h^{(1)}, h^{(2)}\right]\right\rangle=\right. \\
& \quad P_{++}^{3} v_{3}+3 P_{++}^{2} P_{-+} v_{1}+3 P_{++} P_{-+}^{2} v_{-1}+P_{-+}^{3} v_{-3}+
\end{aligned}
$$

$$
\begin{aligned}
& \left(3 P_{++}^{2} P_{+-}+3 P_{++}^{2} P_{--}+6 P_{++} P_{+-} P_{-+}\right) v_{1}+ \\
& \left(3 P_{+-} P_{-+}^{2}+6 P_{+-} P_{-+} P_{--}\right) v_{-1}+3 P_{-+} P_{--}^{2} v_{-3}+ \\
& \left(3 P_{++} P_{+-}^{2}+3 P_{+-}^{2} P_{-+}+6 P_{++} P_{+-} P_{--}\right) v_{-1}+ \\
& \left(3 P_{++} P_{--}^{2}+6 P_{+-} P_{-+} P_{--}\right) v_{-1}+3 P_{-+} P_{--}^{2} v_{-3}+ \\
& \left(P_{+-}^{3}+3 P_{+-}^{2} P_{--}+3 P_{+-} P_{--}^{2}+P_{--}^{3}\right) v_{-3}
\end{aligned}
$$

with

$$
v_{n}=v(n J) .
$$

Equations of motion for the magnetizations $m^{(1)}$ and $m^{(2)}$ as well as for the damage $D$ can be derived by inserting the definitions (14) into the single-site master equation (12). After some manipulations the equations of motion for the magnetizations read

$$
\begin{aligned}
\frac{d}{d t} m^{(n)} & =m^{(n)}\left\{-1+\frac{3}{4}[\tanh (3 J / T)+\tanh (J / T)]\right\} \\
& +\frac{1}{4}\left(m^{(n)}\right)^{3}[\tanh (3 J / T)-3 \tanh (J / T)] .
\end{aligned}
$$

These equations are, of course, identical to the equation of motion of the magnetization derived for a single system within the same framework of statistically independent fluctuations. The point at which the coefficient of the term linear in $m$ on the right-hand side of (17) changes sign defines the (equilibrium) critical temperature $T_{C}$ of the Ising model within our approximation. $T_{C}$ is thus determined by

$$
\frac{3}{4}\left[\tanh \left(3 J / T_{C}\right)+\tanh \left(J / T_{C}\right)\right]=1
$$

which gives $T_{C} / J \approx 2.104$. The stationary solution of (17) can be used to determine the magnetization as a function of temperature. For temperatures $T<T_{C}$ we obtain

$$
\left(m^{(n)}\right)^{2}=\frac{\frac{3}{4}[\tanh (3 J / T)+\tanh (J / T)]-1}{\frac{3}{4} \tanh (J / T)-\frac{1}{4} \tanh (3 J / T)} .
$$

We now turn to the discussion of the Hamming distance $D$. After inserting (14) into (12) the equation of motion of the Hamming distance $D$ can be written as

$$
\begin{aligned}
\frac{d}{d t} D & =(1-D)[W(--\rightarrow+-)+W(--\rightarrow-+)]+ \\
& +D[-1+W(-+\rightarrow+-)+W(-+\rightarrow-+)] .
\end{aligned}
$$

Since in the following we will be mainly interested in the stationary solutions of this equation we restrict the considerations to cases where both systems are in equilibrium at the beginning of the damage spreading process. In doing so we exclude, however, all phenomena connected with the behavior after a quench from high temperatures to temperatures below $T_{C}$. These phenomena require an investigation of the early time behavior and will be analyzed elsewhere [15]. 
It is now useful to distinguish three cases, (i) damage spreading in the paramagnetic phase $\left(T>T_{C}\right)$, (ii) the ferromagnetic phase $\left(T<T_{C}\right)$ where both systems are in the same pure state (i.e., free energy valley), $m^{(1)}=$ $m^{(2)}=m$ and (iii) the ferromagnetic phase $\left(T<T_{C}\right)$ where the two systems are in different pure states, $m^{(1)}=$ $-m^{(2)}=m$.

\section{Paramagnetic phase}

In the paramagnetic phase all $P_{\nu}$ can be expressed in terms of $D$ :

$$
\begin{aligned}
& P_{++}=P_{--}=\frac{1}{2}(1-D), \\
& P_{+-}=P_{-+}=\frac{1}{2} D .
\end{aligned}
$$

By inserting this into the transition probabilities $W(\nu \rightarrow$ $\mu$ ) calculated from (13) and table II the equation of motion (20) of the Hamming distance $D$ can be written as

$$
\frac{d}{d t} D=\frac{1}{2}\left(D-3 D^{2}+2 D^{3}\right) \tanh (3 J / T) .
$$

This equation has three stationary solutions (fixed points), viz. $D_{1}^{*}=0$ which corresponds to both systems being identical, $D_{2}^{*}=1$ where $S^{(1)}=-S^{(2)}$ for all sites and $D_{3}^{*}=1 / 2$ which corresponds to the two systems being completely uncorrelated 16]. To determine the stability of the fixed points we linearize the equation of motion (22) in $d_{k}=D-D_{k}^{*}$. The linearized equation has the solution

$$
d_{k}(t)=d_{k 0} e^{\lambda_{k} t}
$$

with $\lambda_{1}=\lambda_{2}=\frac{1}{2} \tanh (3 J / T)$ and $\lambda_{3}=-\frac{1}{4} \tanh (3 J / T)$. Consequently, the only stable fixed point is $D_{3}^{*}=1 / 2$. In the whole paramagnetic phase the damage spreads and asymptotically reaches the value $D=1 / 2$. If the two systems start very close together ( $D$ small initially) their distance in phase space increases exponentially with a Lyapunov exponent $\lambda_{1}=\frac{1}{2} \tanh (3 J / T)$. Therefore the Glauber dynamics shows chaotic behavior in the whole paramagnetic phase. Note, that for large temperatures the Lyapunov exponent $\lambda_{1}$ goes to zero as $\lambda_{1} \sim 3 J / T$. Thus, the time it takes the system to reach the stationary state $D=D_{3}^{*}=1 / 2$ diverges for $T \rightarrow \infty$. This has recently also been found in simulations $[17]$. The dependence of the Lyapunov exponent on temperature is presented in fig. 1.

\section{Ferromagnetic phase with $m^{(1)}=m^{(2)}=m$}

In this paragraph we study the case where both systems are in the same free energy valley. The single-site probabilities $P_{\nu}$ can be expressed in terms of $D$ and $m$ :

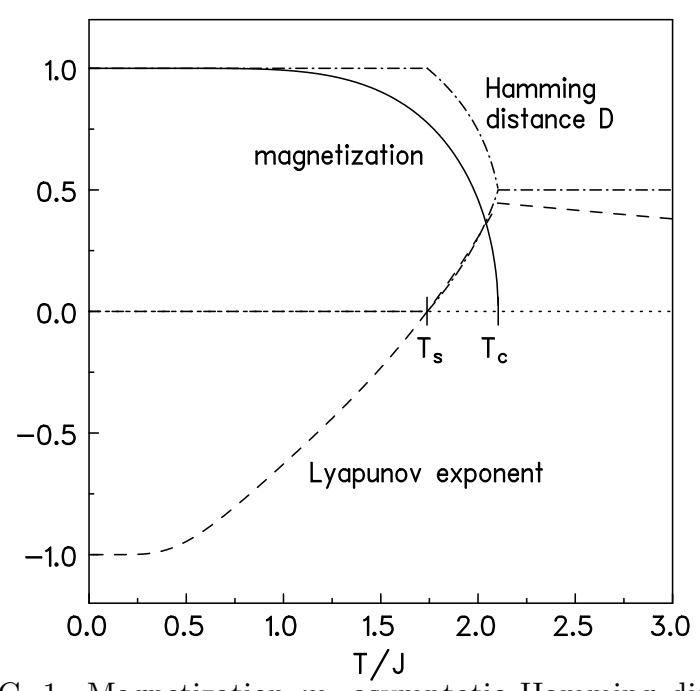

FIG. 1. Magnetization $m$, asymptotic Hamming distance $D^{*}$ and Lyapunov exponent $\lambda_{1}$ as functions of temperature for the Glauber Ising model. Below $T_{c}$ the curve for $D^{*}$ has two branches corresponding to the two systems being in the same or in different free energy valleys.

$$
\begin{aligned}
P_{++} & =\frac{1}{2}(1-D+m), \\
P_{--} & =\frac{1}{2}(1-D-m), \\
P_{+-}=P_{-+} & =\frac{1}{2} D .
\end{aligned}
$$

After inserting this into the averaged transition probabilities (13) the equation of motion of the Hamming distance takes the form

$$
\begin{aligned}
\frac{d}{d t} D= & \frac{1}{2}\left(D-3 D^{2}+2 D^{3}\right) \tanh (3 J / T) \\
- & \frac{3}{4} m^{2}[2 D \tanh (J / T) \\
& \left.\quad-D^{2} \tanh (J / T)+D^{2} \tanh (3 J / T)\right] .
\end{aligned}
$$

This equation has two fixed points $D^{*}$ in the interval $[0,1]$. The first fixed point is $D_{1}^{*}=0$. By linearizing (25) in $d_{1}=D-D_{1}^{*}$ we investigate the stability of this fixed point. We again find that $d_{1}(t)$ follows the exponential law (23) with $\lambda_{1}=\frac{1}{2} \tanh (3 J / T)-\frac{3}{2} m^{2} \tanh (J / T)$. Using for $m^{2}$ the expression (19) it is easy to discuss the behavior of $\lambda_{1}$. For temperatures larger than a spreading temperature $T_{S}$ which is defined by

$$
3 m^{2} \tanh \left(J / T_{S}\right)=\tanh \left(3 J / T_{S}\right)
$$

the Lyapunov exponent $\lambda_{1}$ is positive and thus the fixed point $D_{1}^{*}$ is unstable. For $T<T_{S}$ the Lyapunov exponent $\lambda_{1}$ is negative and the fixed point $D_{1}^{*}$ is stable. Consequently, the Glauber dynamics is chaotic for temperatures above $T_{S}$ but regular below. Eq. (26) gives $T_{S} \approx 1.739 J \approx 0.826 T_{C}$.

For temperatures $T>T_{S}$ the equation of motion (25) possesses another fixed point $D_{3}^{*}$ with $0<D_{3}^{*}<1 / 2$ 
which is always stable. Its temperature dependence is presented in fig. 1. Close to the spreading temperature the asymptotic Hamming distance $D_{3}^{*}$ increases linearly with $T-T_{s}$ which corresponds to the spreading transition being of 2 nd order. The order parameter exponent $\beta$, defined by $D^{*}=\left|T-T_{s}\right|^{\beta}$ is given by $\beta=$. In contrast to the paramagnetic phase, where the two systems eventually become completely uncorrelated, for $T_{s}<T<T_{c}$ the asymptotic Hamming distance $D$ is always smaller than $1 / 2$ so that the two systems remain partially correlated (as it must be the case since both systems are in the same free energy valley). Directly at the spreading point the term linear in $D$ in (25) vanishes. For small Hamming distances the equation of motion now reads $d D / d t \propto-D^{2}$ which gives a power-law behavior $D(t) \propto t^{-\delta}$. The critical exponent is given by $\delta=1$.

\section{Ferromagnetic phase with $m^{(1)}=-m^{(2)}=m$}

We now turn to the case where the two systems are in different free energy valleys. The single-site probabilities $P_{\nu}$ can be expressed in terms of $D$ and $m$ :

$$
\begin{aligned}
P_{++}=P_{--} & =\frac{1}{2}(1-D), \\
P_{+-} & =\frac{1}{2}(D+m), \\
P_{-+} & =\frac{1}{2}(D-m) .
\end{aligned}
$$

With this substitutions the equation of motion (20) of the Hamming distance can be written as

$$
\begin{aligned}
\frac{d}{d t} D= & \frac{1}{2}\left(D-3 D^{2}+2 D^{3}\right) \tanh (3 J / T) \\
+ & \frac{3}{4} m^{2}[\tanh (3 J / T)+\tanh (J / T)-2 D \tanh (3 J / T) \\
& \left.\quad+D^{2} \tanh (3 J / T)-D^{2} \tanh (J / T)\right] .
\end{aligned}
$$

Analogously to the preceeding paragraph, this equation possesses two fixed points. The fixed point $D_{2}^{*}=1$ exits for all temperatures. It is stable for temperatures below $T_{S}$ and unstable above. For $T>T_{S}$ (28) has another fixed point , $D_{4}^{*}$ with $1 / 2<D_{4}^{*}<1$ which is always stable. Its temperature dependence is given in fig. 1.

\section{The limit of high dimensions}

In this subsection we study damage spreading in the Glauber Ising model in the limit of high dimensions, i.e. in the mean-field limit proper. Within the framework of our effective field approach high dimensions correspond to high coordination numbers, i.e. high numbers of nearest neighbors. We therefore consider a Glauber Ising model on a lattice with $z$ nearest neighbors and study the limit $z \rightarrow \infty$. To obtain a physically sensible limit we scale the interaction strength with $z, J=J_{0} / z$.

In the limit $z \rightarrow \infty$ the thermodynamics is described by the usual mean-field theory. The equilibrium critical temperature is given by $T_{C}=J_{0}$ and in the ferromagnetic phase the magnetization is determined by the equation of state

$$
m=\tanh \left(m J_{0} / T\right) .
$$

In order to determine the spreading temperature $T_{S}$ it is sufficient to study the equation of motion (20) of the Hamming distance to linear order in $D$. To this end we have to determine $W(-+\rightarrow+-)$ and $W(-+\rightarrow-+)$ to zeroth order in $D$ but $W(--\rightarrow+-)$ and $W(--\rightarrow-+)$ to linear order in $D$.

To zeroth order in $D$ we have $h^{(1)}=h^{(2)}=h$ and in the limit $z \rightarrow \infty h$ is $\delta$-distributed at $h=J_{0} m$. The transition probabilities are thus given by (see table I)

$$
\begin{aligned}
W(-+\rightarrow+-) & =v\left[-\max \left(-h^{(1)}, h^{(2)}\right)\right] \\
& =v\left(-J_{0}|m|\right), \\
W(-+\rightarrow-+) & =v\left[\min \left(-h^{(1)}, h^{(2)}\right)\right] \\
& =v\left(-J_{0}|m|\right) .
\end{aligned}
$$

We now calculate $W(--\rightarrow+-)$ and $W(--\rightarrow-+)$ to linear order in $D$. These transition probabilities do not have a zeroth-order contribution. In linear order in $D$ only those configurations of the $z$ neighboring sites contribute for which the two systems differ in the state of a single site. In this case $h^{(1)}$ and $h^{(2)}$ differ by $2 J_{0} / z$. Therefore we obtain

$$
\begin{aligned}
W(--\rightarrow+-) & =\Theta\left(h^{(1)}-h^{(2)}\right)\left[v\left(h^{(1)}\right)-v\left(h^{(2)}\right)\right] \\
& =z P_{+-}\left(2 J_{0} / z\right) v^{\prime}\left(J_{0} m\right) .
\end{aligned}
$$

Here $v^{\prime}(h)$ is the derivative of $v$ with respect to its argument. The additional factor $z$ in the second line arises since each of the $z$ neighbors can be the one where the two systems differ. Analogously we obtain

$$
\begin{aligned}
W(--\rightarrow+-) & =\Theta\left(h^{(2)}-h^{(1)}\right)\left[v\left(h^{(2)}\right)-v\left(h^{(1)}\right)\right] \\
& =z P_{-+}\left(2 J_{0} / z\right) v^{\prime}\left(J_{0} m\right) .
\end{aligned}
$$

By inserting these results for the transition probabilities into the equation of motion (20) of the Hamming distance we find

$$
\frac{d}{d t} D=\lambda D
$$

where the Lyapunov exponent $\lambda$ is given by

$$
\begin{aligned}
\lambda= & -1+2 \frac{\exp \left(-J_{0}|m| / T\right)}{\exp \left(J_{0}|m| / T\right)+\exp \left(-J_{0}|m| / T\right)} \\
& +\frac{4 J_{0} / T}{\left[\exp \left(J_{0} m / T\right)+\exp \left(-J_{0} m / T\right)\right]^{2}} .
\end{aligned}
$$

This can be simplified to 


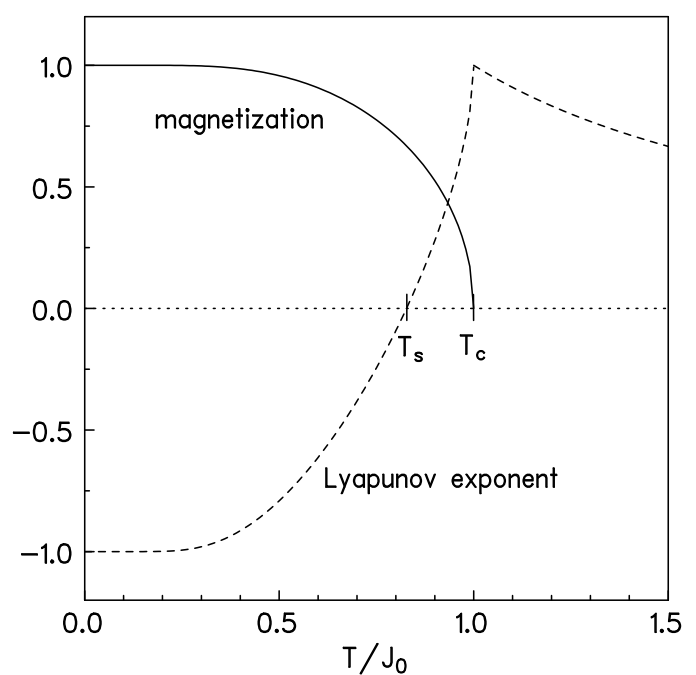

FIG. 2. Magnetization $m$ and Lyapunov exponent $\lambda_{1}$ as functions of temperature for the Glauber Ising model with vanishing external field in the limit of high dimensions.

$$
\lambda=-m+\left(1-m^{2}\right) J_{0} / T \text {. }
$$

In the paramagnetic phase $(m=0)$ the Lyapunov exponent is simply $\lambda=J_{0} / T>0$. Thus the Glauber dynamics is chaotic in the whole paramagnetic phase.

The temperature dependence of the Lyapunov exponent $\lambda$ in the ferromagnetic phase is presented in fig. 2 . $\lambda$ changes sign at $T_{S} \approx 0.827 J_{0}=0.827 T_{C}$. Consequently, the dynamics is chaotic for temperatures larger than $T_{S}=0.827 \mathrm{~J}$ and regular for temperatures smaller than $T_{S}$. Note that the value for $T_{S} / T_{C}$ for the twodimensional model of sec. IIIB is very close to but not identical to the value for the case $z \rightarrow \infty$.

\section{Damage spreading in a field}

In this subsection we generalize the effective-field theory to a finite external magnetic field $h$. For simplicity, we do this only for the model introduced in the preceeding subsection, viz. the limiting case of high dimensions.

The equation of state (29) has to be replaced by

$$
m=\tanh \left[\left(m J_{0}+h\right) / T\right]
$$

Analogously, in all transition probabilities $W(\nu \rightarrow \mu)$ the term $J_{0} m$ has to be replaced by $J_{0} m+h$. After inserting the transition probabilities into the equation of motion (20) of the Hamming distance one finds $d / d t D=\lambda D$ and the Lyapunov exponent can again be expressed in terms of the magnetization:

$$
\lambda=-m+\left(1-m^{2}\right) J_{0} / T .
$$

The temperature and field dependence of the Lyapunov exponent is illustrated in fig. 3. Obviously, the exter-

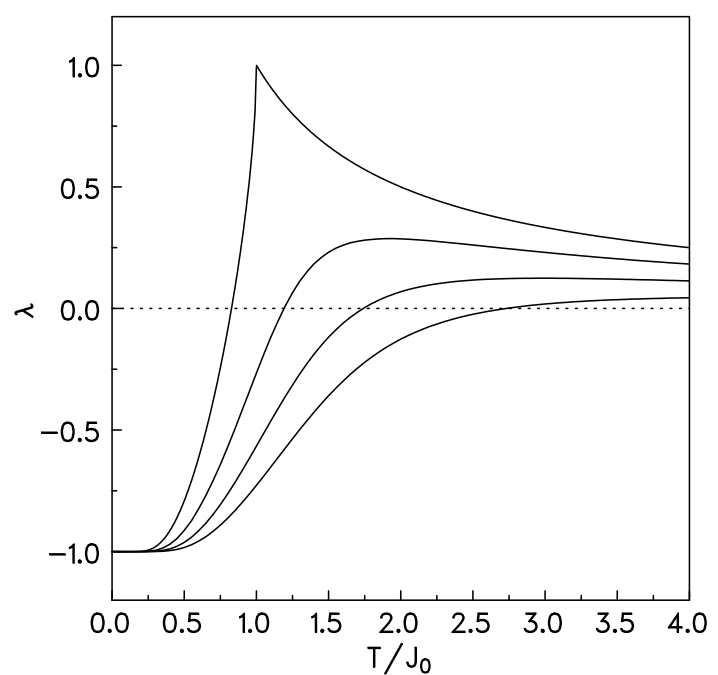

FIG. 3. Lyapunov exponent $\lambda$ as a function of temperature for external fields $h=0,0.2,0.4$ and 0.6 (from up to down).

nal field shifts the spreading temperature to higher values, thus suppressing chaotic behavior and stabilizing the regular phase. The phase boundary between the chaotic and the regular phase can be easily determined by solving the equation $\lambda=0$. The resulting phase diagram is presented in fig. 4. For comparison we also give simulation results [18] for a three-dimensional Glauber Ising model. An investigation of (35) and (36) for large temperatures shows that the spreading temperature $T_{S}(h)$ diverges for $h / J_{0} \rightarrow 1$ as

$$
T_{S}(h) / J_{0}=1 /\left(1-h / J_{0}\right) \quad \text { for } h / J_{0} \rightarrow 1 .
$$

Consequently, for external fields $h>J_{0}$ the dynamics is always regular.

\section{CONCLUSIONS}

To summarize, we have developed a master equation approach to damage spreading and applied it to the Glauber Ising model. The master equation is an exact description of the damage spreading problem, it does not contain any approximations. We have then solved the master equation within an effective-field theory for various physical situations.

In this final section we discuss some aspects which have not been covered yet. First, we compare the results of our effective-field theory with numerical simulations of damage spreading of the Glauber Ising model in two and three dimensions [9, 11, 18]. In agreement with the simulation results we find a spreading transition below the equilibrium critical temperature of the Ising model. Our mean-field value $T_{s} / T_{c} \approx 0.827$ is considerably lower than the latest numerical values [11] of 0.992 for a twodimensional and 0.922 for a three-dimensional Glauber Ising model. We expect our value to be exact, however, for an infinite-dimensional model or, equivalently, 


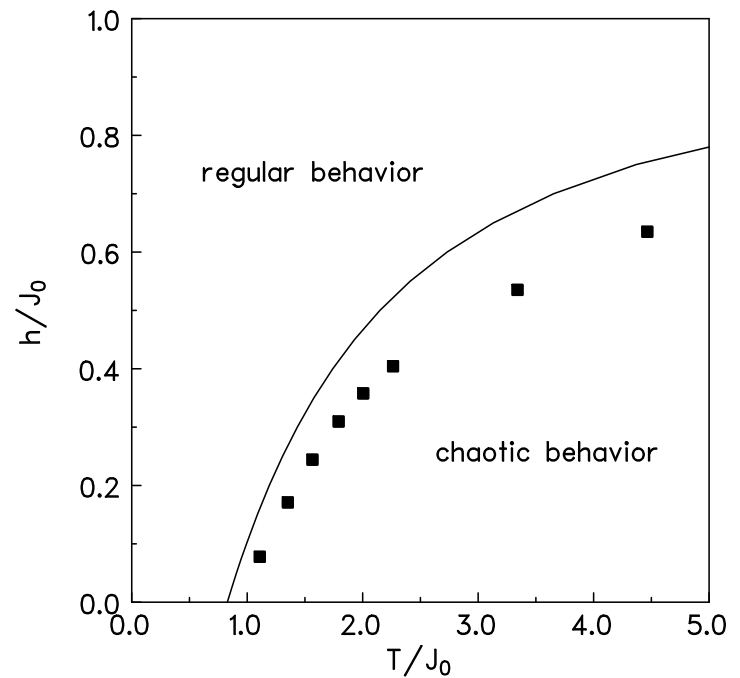

FIG. 4. Phase diagram of damage spreading in the Glauber Ising model in the limit of infinite dimensions. The full line shows the result of our theory, the squares are simulation results of Le Caër with $T$ and $h$ rescaled by $T_{C}$.

for infinite range of the interaction. Grassberger 10 conjectured that the damage spreading transition in the Glauber Ising model is in the universality class of directed percolation. Our results are compatible with that, since the values of the critical exponents $\beta$ (which describes the dependence of the stationary damage on the reduced temperature) and $\delta$ (which describes the time decay of the damage at the spreading temperature) are identical to the mean-field values $\beta=\delta=1$ of directed percolation.

Second, we want to clarify the relation to damage spreading in an Ising model with heat-bath dynamics. As already discussed in the introduction the heat-bath Ising model does not show any spreading of damage within a single pure state (free energy valley). When applying our effective-field theory to the heat-bath Ising model we find [14] only a single fixed point $D_{1}^{*}=0$ if both systems are in the same pure state [19]. If the two systems are in different pure states (for $T<T_{C}$ ) we also find a single fixed point only, viz $D_{2}^{*}=m$. Thus there is no chaotic behavior within one pure state. However, the damage can spread (or, at least, will not heal) in the heat-bath Ising model if the two copies start in different pure states or choose to develop into different pure states after a quench from high temperatures. For this case a mean-field theory similar to our's has been considered before [20].

Finally, we discuss possible extensions of the present theory. In principle, the master equation approach of sec. II can be applied to any damage spreading problem in which the dynamics of a single system is given by a stochastic map as in (2) (or a more general map that involves several sites in each time step). It would be very interesting to remap the master equation onto a field theory and then apply renormalization group methods to determine the critical behavior.
An obvious idea is to include quenched disorder into the Hamiltonian of the Ising model either in the form of a random external field or in the form of random interactions. Such systems have been numerically investigated in some detail, in particular in the case of random interactions [21]. Recently some interesting results have also been achieved for random fields [17]. Some investigations on the application of the master equation approach to disordered systems are in progress.

\section{ACKNOWLEDGMENTS}

This work was supported in part by the DFG under grant number Vo 659/1-1 and by the NSF under grant number DMR-95-10185.

[1] P. C. Hohenberg and B. I. Halperin, Rev. Mod. Phys. 49, 435 (1977).

[2] J. S. Langer in C. Godreche (Ed.) Solids far from equilibrium, Cambridge University Press, Cambridge (1992), p297.

[3] A. Kauffman, J. Theor. Biol. 22, 437 (1969).

[4] B. Derrida and Y. Pomeau, Europhys. Lett. 1, 45 (1986); B. Derrida and G. Weisbuch, Europhys. Lett. 4, 657 (1987).

[5] H.E. Stanley, D. Stauffer, J. Kertesz, and H.J. Herrmann, Phys. Rev. Lett. 59, 2326 (1987).

[6] see, .e.g., H. G. Schuster, Deterministic chaos, VCH Publishers, Weinheim (1984).

[7] A. Coniglio, L. de Arcangelis, H.J. Herrmann, and N. Jan, Europhys. Lett. 8, 315 (1989).

[8] S.C. Glotzer, P.H. Poole, and N. Jan, J. Stat. Phys. 68, 895 (1992).

[9] U. Costa, J. Phys. A 20, L583 (1987); G. Le Caër, Physica A 159, 329 (1989).

[10] P. Grassberger, J. Stat. Phys. 79, 13 (1995).

[11] P. Grassberger, J. Phys. A 28, L67 (1995).

[12] P.Grassberger, Physica A 214, 547 (1995).

[13] F. Wang, N. Hatano and M. Suzuki, J. Phys. A 28, 4545 (1995);

F. Wang and M. Suzuki, Physica A 223, 34 (1996).

[14] T. Vojta, cond-mat/961084, J. Phys. A, in press.

[15] T. Vojta, to be published.

[16] For vanishing external field the Glauber algorithm is invariant under a global flip of all spins. Thus, the existence of $D_{1}^{*}=0$ automatically implies the existence another fixed point, $D_{2}^{*}=1$, with the same stability properties.

[17] T. Wappler, T. Vojta, and M. Schreiber, condmat/961089, submitted to Phys. Rev. B.

[18] G. Le Caër, J. Phys. A 22, L647 (1989).

[19] In contrast to the Glauber dynamics the heat-bath algorithm does not preserve the symmetry with respect to a 
global flip of all spins. Therefore $D=1$ is not a fixed point here.

[20] O. Gollinelli and B. Derrida, J. Phys. (Paris) 49, 1663 (1988).

[21] see, e.g. B. Derrida, Phys. Rep. 184, 207 (1989); I.A. Campbell, Europhys. Lett. 21, 959 (1993); F.A. Tamarit and E.M. Fleury Curado, J. Phys. A 27, 671 (1994). 